\title{
EFFECTS OF PARTICLE MIXING AND SCATTERING IN THE DUSTY GAS FLOW THROUGH MOVING AND STATIONARY CASCADES OF AIRFOILS
}

\section{Yu. M. Tsirkunov, D. A. Romanyuk, and S. V. Panfilov}

Faculty of Aerospace Engineering Baltic State Technical University 1st Krasnoarmeiskaya Str. 1, St. Petersburg 190005, Russia

Time-dependent two-dimensional (2D) flow of dusty gas through a set of two cascades of airfoils (blades) has been studied numerically. The first cascade was assumed to move (rotor) and the second one to be immovable (stator). Such a flow can be considered, in some sense, as a flow in the inlet stage of a turbomachine, for example, in the inlet compressor of an aircraft turbojet engine. Dust particle concentration was assumed to be very low, so that the interparticle collisions and the effect of the dispersed phase on the carrier gas were negligible. Flow of the carrier gas was described by full Navier-Stokes equations. In calculations of particle motion, the particles were considered as solid spheres. The particle drag force, transverse Magnus force, and damping torque were taken into account in the model of gas-particle interaction. The impact interaction of particles with blades was considered as frictional and partly elastic. The effects of particle size distribution and particle scattering in the course of particle-blade collisions were investigated. Flow fields of the carrier gas and flow patterns of the particle phase were obtained and discussed.

\section{INTRODUCTION}

In aircraft turbojet engines and other axial turbomachines, in practice, a working gas flowing through a machine duct can contain suspended solid particles or liquid droplets. The presence of such a dispersed phase in the flow results in some new effects which are usually undesirable. In particular, it causes the erosion of blades due to multiple impacts of particles or droplets and additional momentum

This is an Open Access article distributed under the terms of the Creative Commons Attribution-Noncommercial License 3.0, which permits unrestricted use, distribution, and reproduction in any noncommercial medium, provided the original work is properly cited. 


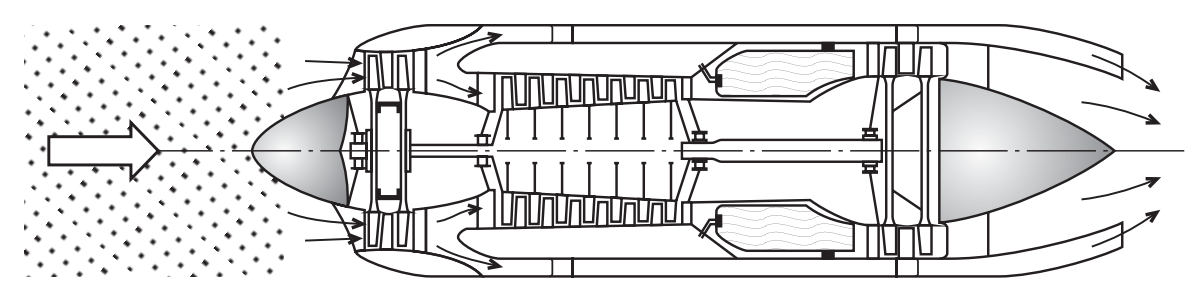

Figure 1 Dusty gas flow through an aircraft turbojet engine

and energy losses [1]. In view of it, one of important problems is the protection of blades and other parts of the duct from erosion. The most vulnerable to the effect of particles is the inlet stage of a turbomachine. In application to the aircraft turbojet engine (Fig. 1), it is the inlet compressor. For predicting the regions of blade surfaces exposed to the strongest erosive effect, there is a need of having a clear insight into characteristic features of particle behavior in the flow. The flow of the carrier gas in the turbomachine duct is strictly speaking threedimensional (3D). However, theoretical estimates for inertial forces due to flow swirling by rotor blades indicate that in channels of axial turbomachines these forces are negligible [2]. Such estimates are in good agreement with experiments everywhere in the duct except for a thin region adjacent to the walls. Therefore, many of the important flow features in the inlet stage and in the sequential rims can be studied using a 2D flow model in the plane, which represents a developed mean-circle cross section (Fig. 2). Such an approach turned out very fruitful and led to the development of the well-known 2D theory of cascade flow (see,
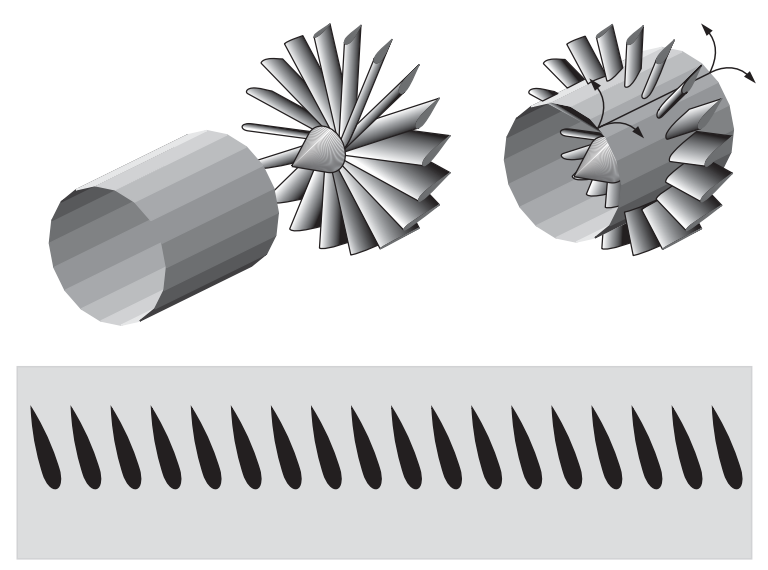

Figure 2 Schematic of transition from the 3D configuration of a rim of blades to the $2 \mathrm{D}$ configuration of a cascade of airfoils 
e.g., [2]). This theory deals with steady-state dust-free gas flows. The steadystate flow of gas-particle mixture through an immovable cascade was analyzed by Hussein and Tabakoff [3]. Steady-state gas-particle flow approximation was used in [4] to study the flow in a single rotating cascade.

In the present study, the behavior of solid particles in the time-dependent high-speed subsonic 2D flow in a set of two, one moving and the other stationary, cascades of blades was simulated numerically and analyzed. The particle mass load was assumed to be very low, so that the interparticle collisions and the effect of the dispersed phase on the carrier gas flow could be neglected. In this case, the problem of two-phase flow simulation was reduced to the sequential solving of two problems:

(i) computation of the carrier gas flow field; and

(ii) calculation of particle motion in this flow field.

The input data in the calculations (flow properties, speed of the moving cascade, airfoil size, etc.) were taken close to those in the flow through the axial compressor of an aircraft turbojet engine. The main objective of this study was to understand how such effects as the particle size distribution and the particle scattering in the course of particle--wall collisions influence the particle-phase flow structure in the complex flow of dusty gas through the inlet rotor-stator system of a turbojet engine.

\section{PROBLEM FORMULATION AND NUMERICAL METHOD}

\subsection{Flow Schematic}

Consider a two-phase gas-particle flow through a set of two cascades with the first moving at constant velocity $V_{r}$ and the second at rest (Fig. 3). Both cas-

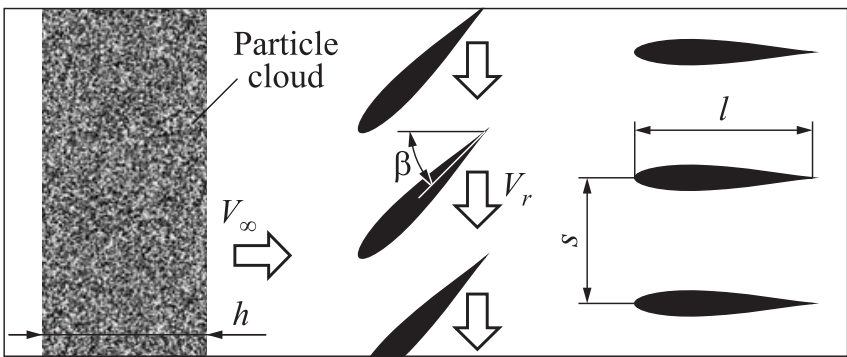

Figure 3 Arrangement of two cascades in undisturbed flow 
cades have the same pitch $s$ (distance between airfoils along the cascade). The airfoils of the first cascade are set at angle $\beta$ with respect to the undisturbed flow direction. For visualizing the particle-phase flow in the computational simulation, a cloud of particles of finite width $h$ equal to the airfoil chord $l$ was considered.

Under the above assumptions, a two-phase flow can be considered as a oneway coupled and dilute. The estimates for the particle concentration required for this model to be valid were obtained in [5]. The upper bound of the concentration was shown to depend on the particle size, all other parameters being fixed. The motion of particles is governed by the gas-particle interaction and particle-airfoil collisions. As the particles are more inertial than the carrier gas they do not follow the streamlines and can collide with airfoils and rebound from them. The trajectories of rebounded (reflected) particles can intersect with each other and with the trajectories of incident particles. Calculations of a large number of particles allow the specific features of particle behavior in the flow to be better understood. As the gas-particle flow considered in this study is time-dependent, the instantaneous flow patterns of the carrier gas and particle phase are presented and discussed.

\subsection{Modeling of Carrier Gas Flow}

The classical theory of cascades is based on the model of inviscid gas. Preliminary computational simulations of a high-speed subsonic gas flow through the rotor-stator set of cascades was performed on the basis of Euler equations and full Navier-Stokes equations. The same computational grid was used in the computation domain in both cases. Comparison of the results showed that the effects associated with gas viscosity (development of boundary layers on airfoils and vortex wakes behind them) played an important role in forming the flow structure. The Reynolds number $\operatorname{Re}_{\infty}=\rho_{\infty} V_{\infty} l / \mu_{\infty}$ in the problem under consideration is approximately $1.4 \cdot 10^{6}$, i. e., the flow is turbulent and, in this case, the conventional approach requires the use of Reynolds averaged Navier-Stokes equations instead of Navier-Stokes equations themselves. However, this approach is shared here only in part. The matter is that the large-scale vortex flow structure, which is of great importance in many applications, can be obtained very often without fine modeling of turbulence and even without boundary layer effects. In the present study, a large-scale vortex street arises behind airfoils due to the interaction of boundary layers running off airfoils. This effect will be best demonstrated by the computational results in section 3 .

Navier-Stokes equations for the time-depended compressible 2D flow can be written in Cartesian coordinates $(x, y)$ in the following compact form [6]:

$$
\frac{\partial \mathbf{U}}{\partial t}+\frac{\partial \mathbf{F}_{x}}{\partial x}+\frac{\partial \mathbf{F}_{y}}{\partial y}=\frac{\partial \mathbf{G}_{x}}{\partial x}+\frac{\partial \mathbf{G}_{y}}{\partial y}
$$


where $\mathbf{U}, \mathbf{F}_{x}, \mathbf{F}_{y}, \mathbf{G}_{x}$, and $\mathbf{G}_{y}$ vectors are defined as follows:

$$
\left.\begin{array}{r}
\mathbf{U}=\left(\begin{array}{c}
\rho \\
\rho u \\
\rho v \\
\rho e
\end{array}\right) ; \quad \mathbf{F}_{x}=\left(\begin{array}{c}
\rho u \\
\rho u^{2}+p \\
\rho u v \\
(\rho e+p) u
\end{array}\right) ; \quad \mathbf{F}_{y}=\left(\begin{array}{c}
\rho v \\
\rho u v \\
\rho v^{2}+p \\
(\rho e+p) v
\end{array}\right) ; \\
\mathbf{G}_{x}=\left(\begin{array}{c}
0 \\
0 \\
\tau_{x y} \\
\tau_{y y} \\
\tau_{x y} \\
u \tau_{x x}+v \tau_{x y}-q_{x}
\end{array}\right) ; \quad \mathbf{G}_{y}=\left(\begin{array}{c} 
\\
u \tau_{x y}+v \tau_{y y}-q_{y}
\end{array}\right) \cdot
\end{array}\right\}
$$

Here,

$$
\begin{gathered}
p=\rho R T ; \quad e=c_{V} T+\frac{u^{2}+v^{2}}{2} ; \quad q_{x}=-\lambda \frac{\partial T}{\partial x} ; \quad q_{y}=-\lambda \frac{\partial T}{\partial y} ; \\
\tau_{x x}=\frac{2}{3} \mu\left(2 \frac{\partial u}{\partial x}-\frac{\partial v}{\partial y}\right) ; \quad \tau_{y y}=\frac{2}{3} \mu\left(2 \frac{\partial v}{\partial y}-\frac{\partial u}{\partial x}\right) ; \quad \tau_{x y}=\mu\left(\frac{\partial u}{\partial y}+\frac{\partial v}{\partial x}\right) .
\end{gathered}
$$

In the above equations, $t$ is the time; $\rho, p, e, T, \mu$, and $\lambda$ are the gas density, pressure, specific total energy, temperature, viscosity, and thermal conductivity, respectively; $u$ and $v$ are the $x$ - and $y$-components of the velocity vector; $R$ is the gas constant; and $c_{V}$ is the specific heat at constant volume. In the present study, the following relations for $\mu$ and $\lambda$ were used:

$$
\mu=\mu_{0}\left(\frac{T}{T_{0}}\right)^{3 / 2} \frac{T_{0}+S_{0}}{T+S_{0}} ; \quad \lambda=\frac{c_{p} \mu}{\operatorname{Pr}}
$$

where the first relation is the Sutherland formula: $\mu_{0}=1.71 \cdot 10^{-5} \mathrm{~N} \cdot \mathrm{s} / \mathrm{m}^{2}$, $T_{0}=288 \mathrm{~K}, S_{0}=117 \mathrm{~K} ; \operatorname{Pr}$ is the Prandtl number; and $c_{p}$ is the specific heat at constant pressure.

The governing equations were solved numerically. The conditions at the boundaries of the computation domain will be described and discussed in subsection 2.4 .

\subsection{Modeling of Particle-Phase Flow}

The Lagrangian approach was used for modeling the motion of particles. The motion of a particle was described by the momentum and angular momentum equations which were supplemented with the kinematic relation between the particle position vector $\mathbf{r}$ and velocity vector $\mathbf{v}_{\mathrm{p}}$ :

$$
m_{p} \frac{d \mathbf{v}_{p}}{d t}=\mathbf{f}_{D}+\mathbf{f}_{\mathrm{M}} ; \quad J_{p} \frac{d \boldsymbol{\omega}_{p}}{d t}=\mathbf{L}_{p} ; \quad \frac{d \mathbf{r}}{d t}=\mathbf{v}_{p}
$$


Here, $m_{p}=4 \rho_{p} \pi r_{p}^{3} / 3, J_{p}=2 m_{p} r_{p}^{2} / 5, r_{p}$, and $\boldsymbol{\omega}_{p}$ are the particle mass, moment of inertia, radius, and rotation velocity, respectively. The force acting on a particle in the gas flow includes the drag force $\mathbf{f}_{D}$ and lift Magnus force $\mathbf{f}_{\mathrm{M}}$, which far exceed all other force components. The Magnus force is developed due to simultaneous translational and rotational motion of a particle. It can be significant for particles twisted in particle-airfoil collisions. The damping torque $\mathbf{L}_{p}$ acts on a particle if its relative rotational velocity is nonzero. For calculating $\mathbf{f}_{D}, \mathbf{f}_{\mathrm{M}}$, and $\mathbf{L}_{p}$, the conventional relations were used:

$$
\begin{aligned}
\mathbf{f}_{D} & =\frac{1}{2} C_{D} \pi r_{p}^{2} \rho\left|\mathbf{v}-\mathbf{v}_{p}\right|\left(\mathbf{v}-\mathbf{v}_{p}\right) ; \\
\mathbf{f}_{\mathrm{M}} & =\frac{4}{3} C_{\boldsymbol{\omega}} \pi r_{p}^{3} \rho\left[\left(\boldsymbol{\omega}-\boldsymbol{\omega}_{p}\right) \times\left(\mathbf{v}-\mathbf{v}_{p}\right)\right] ; \\
\mathbf{L}_{p} & =\frac{1}{2} C_{L} r_{p}^{5} \rho\left|\boldsymbol{\omega}-\boldsymbol{\omega}_{p}\right|\left(\boldsymbol{\omega}-\boldsymbol{\omega}_{p}\right), \quad \boldsymbol{\omega}=\frac{1}{2} \operatorname{curl} \mathbf{v} .
\end{aligned}
$$

Coefficients $C_{D}, C_{\omega}$, and $C_{L}$ depend on the dimensionless parameters of the flow around a particle: relative Mach and Reynolds numbers, relative rotational Reynolds number, etc. These coefficients were calculated using approximate formulae obtained on the basis of theoretical solutions, numerical results, and experimental data. For $C_{D}$, Henderson relations [7] were used. For calculating $C_{\omega}$, the exact solution obtained by Rubinow and Keller [8] was used as well as the formula suggested by Oesterlé and Bui Dinh [9]. The expression for $C_{L}$ was taken in the form suggested by Dennis et al. [10].

For determining particle parameters just after particle rebounding from the airfoil surface, the semiempirical model [11] for spherical particles and the simplified model with constant restitution coefficients for the normal and tangential components of the contact point velocity [12] for nonspherical particles were used. The semiempirical model was based on the laws of mechanics and experimental data for the restitution coefficients of the translational velocity components.

In the majority of papers on two-phase gas-particle flow, the particles are assumed to be rigid spheres of the same radius and scattering in particle-wall collisions is not taken into account. However, in practice, particles are distributed in size that results in mixing of particles of different size in the disturbed flow. Moreover, the particle shape is often different from spherical, and this results in particle scattering in the course of particle-wall collisions. In the present study, the behavior of polydisperse particles with log-normal size distribution in undisturbed flow is considered. Also, the scattering of particles due to their nonspherical shape is taken into account. In the case of log-normal size distribution, the particle mass distribution function takes the form: 


$$
g_{\infty}\left(r_{p}\right)=\frac{1}{\sqrt{2 \pi} r_{p} \log \sigma} \exp \left[-\left(\frac{\log r_{p}-\log r_{g}}{\sqrt{2} \log \sigma}\right)^{2}\right]
$$

where parameter $r_{g}$ was correlated with the most probable particle size $r_{\text {pm }}$ by

$$
r_{g}=r_{\mathrm{pm}} \exp \left(\log ^{2} \sigma\right)
$$

Figure 4 shows the plot of function (4) for $\sigma=1.2$. For investigating the effect of particle scattering in the model of particle-wall collisions, particles with the shape of ellipsoid of revolution were considered (Fig. 5). The ratio of axes $b / a$ ( $a$ is the axis in

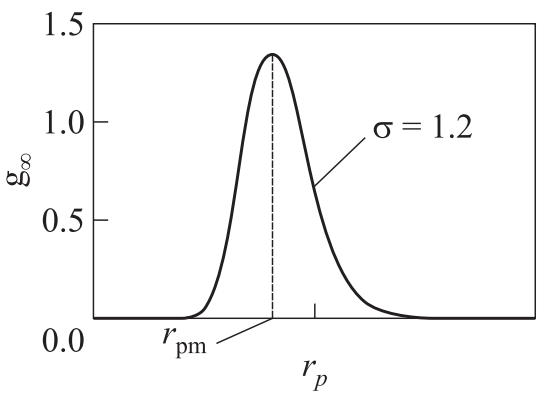

Figure 4 Log-normal distribution $\xi$-direction, and $b$ is the axis in $\eta$ - and $\zeta$-direction) is taken close to unity ( 0.8 and 1.25$)$, so that the particle shape is close to spherical. Collision of a nonspherical particle with a wall is $3 \mathrm{D}$. The model of such collisions and the scattering properties for particles of different shape were described in $[12,13]$. In the computational simulations of particlewall collision in the present study, the particle orientation in space just before collision was considered as random (the orientations of axes $\xi, \eta$, and $\zeta$ relative to $x, y$, and $z$ were equiprobable). Just after collision, a particle had three components of both translational and rotational velocities. However, when simulating the particle-phase flow, the reflected particles were considered as spherical and their scattering only in $(x, y)$-plane was taken into account. Such an approach

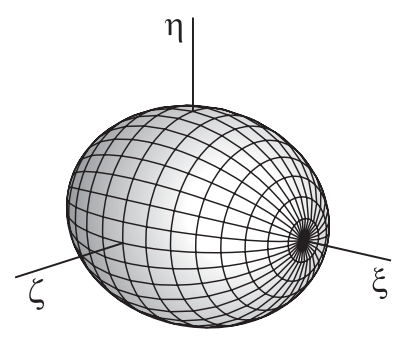

(a)

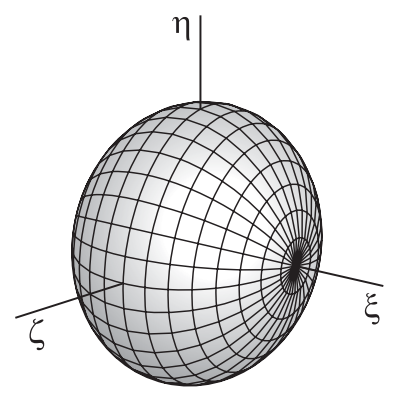

(b)

Figure 5 Ellipsoidal particle shape when modeling its scattering due to particleairfoil collision: prolate $(a)$ and oblate $(b)$ ellipsoids of revolution 
allowed understanding whether the effect of scattering was important or not, but the question of validation of the flow model remained open.

\subsection{Numerical Method}

The computational domain consisted of two blocks (Fig. 6). The left block moved together with a rotor blade, and the right block was at rest. The structured curvilinear grid fitted to the blade contours was introduced in each block. Both grids were refined near the blade surfaces and in the regions behind blades to resolve the flow structure inside the boundary layers and in the wakes. The length of the computational domain in the main flow direction was $4.4 l$.

A finite-volume explicit in time method was used for the numerical solution of Navier-Stokes equation (1). Inviscid fluxes $\mathbf{F}_{x}$ and $\mathbf{F}_{y}$ of conservative variables $\mathbf{U}$ (see Eqs. (2)) through cell faces were calculated by the Roe scheme [14] with the use of entropy correction suggested by Yee and Harten [15]. For calculating viscous fluxes $\mathbf{G}_{x}$ and $\mathbf{G}_{y}$, the reconstruction of gas parameters on the grid with taking into account their gradients inside every cell was used. The method applied was of the second order in space. The time step was chosen from the conventional stability condition.

The periodic conditions in $y$-direction were set at the top and bottom boundaries of each block. This was possible if no large-scale regions of separated flow arose. A special matching procedure was used at the contact boundary between the moving and quiescent blocks [16]. At the inlet boundary of the moving block, the velocity and density calculated from the given total enthalpy and entropy in the undisturbed flow were specified, and the pressure was extrapolated from the computational domain. At the outlet boundary of the quiescent block, the pressure was taken equal to $1.2 p_{\infty}$ (this value agreed with some

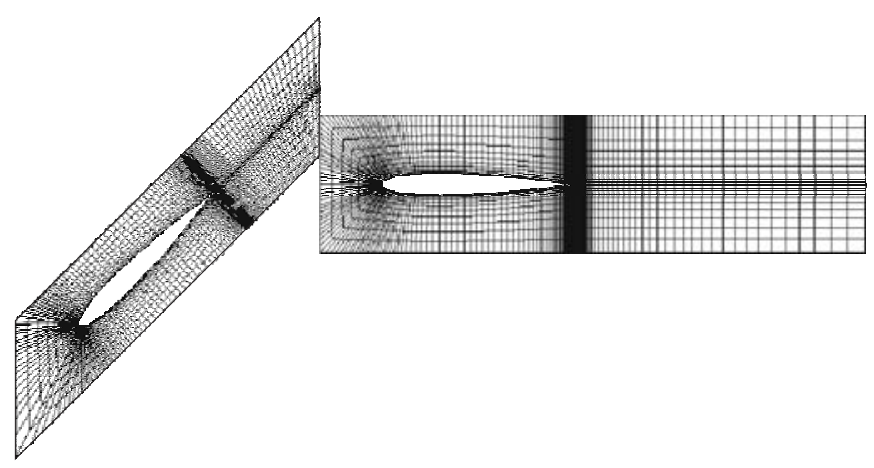

Figure 6 Schematic of the computational domain and grid 
experiments) and other parameters were extrapolated from the computational domain. Such a technique was in agreement with the characteristic properties of gas dynamic equations. At the airfoil surface, the no-slip condition and the constant-temperature wall condition were imposed.

At $t=0$, a uniform flow with undisturbed parameters was taken as the initial condition. Then, the computational simulation of the carrier gas flow was started and continued up to a quasi-time-periodic solution was reached. Thereafter, the particles were introduced into the flow.

Equations (3) for particles were solved by a predictor-corrector method of the second order accuracy. The calculations were carried out simultaneously with the continuing solution of Navier-Stokes equations. The total number of particles in a cloud was taken equal to 50,000 yielding a high resolution of the particle-phase flow structure when visualizing the computational results.

\section{RESULTS AND DISCUSSION}

\subsection{Input Data}

The parameters of cascades and flow properties were taken close to those typical for the inlet compressor of a turbojet engine. The chord of airfoils was equal to $l=10 \mathrm{~cm}$, cascade pitch $s=7 \mathrm{~cm}$, angle of attack $\beta=45^{\circ}$, the profile of blades was NACA0012, main (undisturbed) flow velocity $V_{\infty}=200 \mathrm{~m} / \mathrm{s}$, cascade velocity $V_{r}=150 \mathrm{~m} / \mathrm{s}$, the carrier gas was air $(R=287 \mathrm{~J} /(\mathrm{kg} \cdot \mathrm{K})$, and $\left.c_{p} / c_{V}=1.4\right)$, the Prandtl number $\operatorname{Pr}=0.72$, flow density $\rho_{\infty}=1.21 \mathrm{~kg} / \mathrm{m}^{3}$, and temperature $T_{\infty}=288 \mathrm{~K}$. These values corresponded to flow Mach number of $\mathrm{M}_{\infty}=0.59$. The particle and blade materials were $\mathrm{SiO}_{2}$ and steel, respectively.

\subsection{Results for Carrier Gas}

The majority of calculations for the carrier-gas flow field were performed using Navier-Stokes equations. However, for comparison and for estimating the numerical viscosity, some calculations were also made using Euler equation with the same grids. Thus, the flow fields were obtained for both cases.

Figure 7 shows the results for the entropy function $\theta=p / \rho^{\gamma}$. It is clearly seen that Euler equations provide nearly homogeneous entropy field (Fig. $7 a$ ) which is in good agreement with the theoretical statement that the entropy in the flow of ideal gas remains constant. In the flow field of viscous gas (Fig. $7 b$ ), the spots with much higher entropy than in the main stream are evident. These inhomogeneities arise due to entropy production in boundary layers at blade 

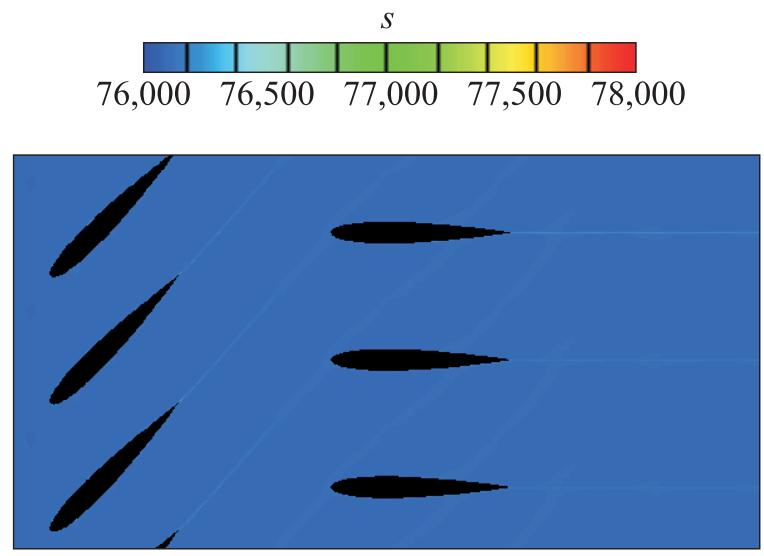

(a)

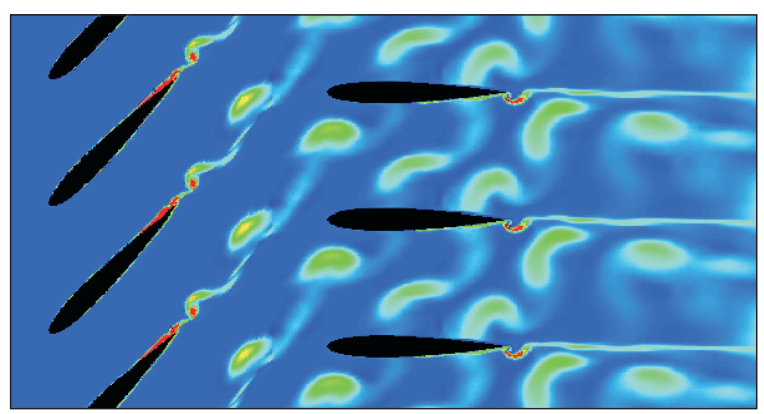

(b)

Figure 7 Instantaneous fields of entropy function $\theta=p / \rho^{\gamma}$ in the carrier gas flow: calculations based on Euler $(a)$ and Navier-Stokes $(b)$ equations. (Refer Tsirkunov et al., p. 468.)

surfaces and inside separated eddies in vortex wakes behind blades. The structure of wakes is similar to that of the von Karman vortex street. The flow in both cases was subsonic in the stationary system of coordinates. At the outlet boundary of the computational domain (right boundary of the quiescent grid block), the gas velocity, density, and temperature reach nearly constant values: $V=115 \mathrm{~m} / \mathrm{s}$, $\rho=1.56 \mathrm{~kg} / \mathrm{m}^{3}$, and $T=304 \mathrm{~K}$. These parameters correspond to the flow Mach number of $\mathrm{M}=0.3$.

The computational experiments have shown that the numerical solution of Euler equations reached with time a periodic behavior illustrated in Fig. $8 a$. In this figure, $\tau=t / t_{s}$ where $t_{s}=s / V_{r}\left(t_{s}=0.47 \cdot 10^{-3} \mathrm{~s}\right.$ for the given values of $s$ and $V_{r}$ ). The period includes three maxima and three minima. Figure $8 b$ shows 


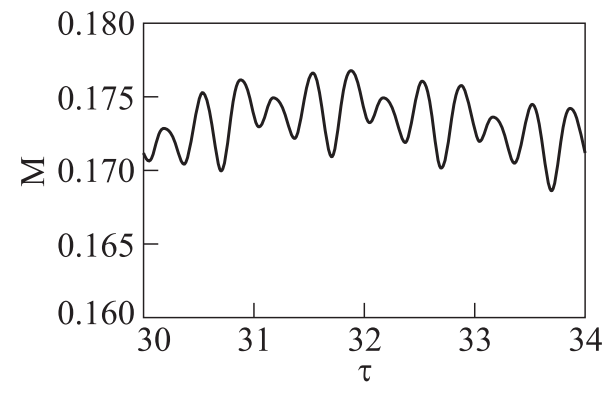

(a)

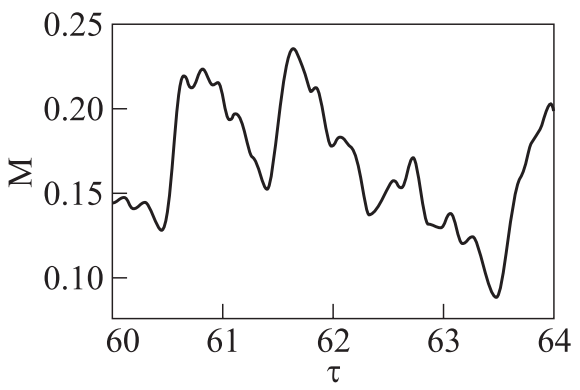

(b)

Figure 8 Time histories of the Mach number upstream of a stator airfoil: calculations based on Euler $(a)$ and Navier-Stokes $(b)$ equations

the time-dependent solution obtained with Navier-Stokes equations. It is seen that in this case, the time-periodic solution is not reached. Such a behavior is caused by two independent time-periodic processes: separation of eddies from blades, and motion of spatially periodic rotor blades relative to the stator blades with the same spatial period. The first process is determined by the Reynolds number of the flow around a blade, whereas the second is determined by velocity $V_{r}$ and the cascade pitch $s$. If the periods of both processes are not multiple, their interaction can result in the "stochastic" behavior of flow parameters in time.

\subsection{Results for the Particle Phase}

For visualizing the particle-phase flow, consider a particle cloud of finite width $h$ in the undisturbed flow (see Fig. 1). In the calculations, $h$ was taken equal to $l$. The particles in the cloud were distributed in space randomly to get a uniform distribution. The results discussed below were obtained for the carrier gas flow field computed using Navier-Stokes equations. The instantaneous particle-flow patterns at a certain (fixed) time are shown in Figs. 9-11. Particle radii of $r_{p}=5,10$, and $20 \mu \mathrm{m}$ corresponded to the Stokes number St $=1.70$, 6.82 , and 27.28, respectively. The Stokes number was defined as the ratio of the particle dynamic relaxation length (with the use of the Stokes law for the particle drag force) to the characteristic length in the flow $l$ :

$$
\mathrm{St}=\frac{2 \rho_{p} r_{p}^{2} V_{\infty}}{9 \mu_{\infty} l} .
$$

Figure 9 shows the dependence of the flow structure of monodisperse spherical particles on particle radius. A cloud is seen to stretch in the flow and the 


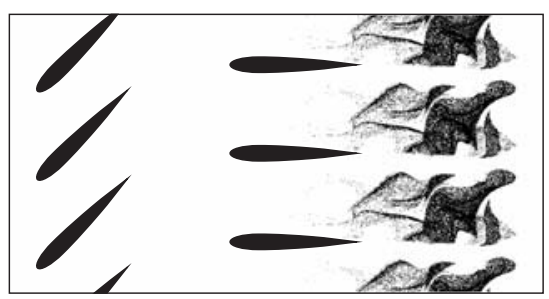

(a)

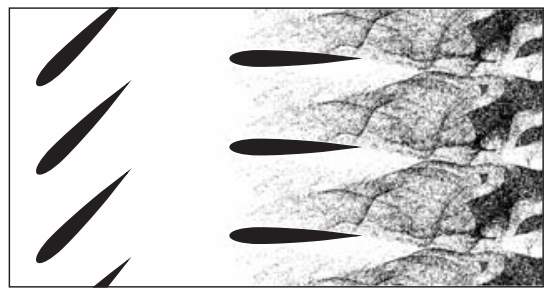

(b)

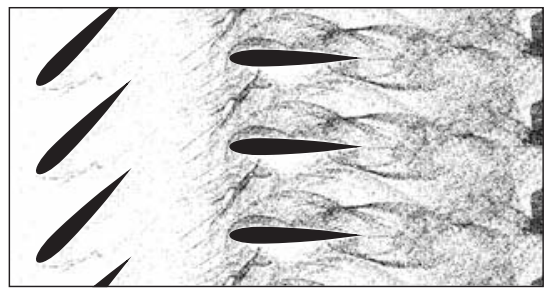

(c)

Figure 9 Instantaneous flow patterns of monodisperse spherical particles depending on the particle radius: $r_{p}=5(a) ; 10(b)$, and $20 \mu \mathrm{m}(c)$; a semiempirical model of particle-wall collisions [11] is used

distribution of particles in space becomes strongly nonuniform. Narrow layers with high particle concentration are clearly visible. Such redistribution of particles occurs due to the action of nonuniform flow field of the carrier gas. For small particles $(\mathrm{St}<10$ ), the effect of boundary layers and vortex wakes is of great importance in the process of redistribution. For large particles (St > 20), this effect is negligible, but the particle-blade collisions play a key role.

Instantaneous patterns of spherical particles with log-normal size distribution in an initial cloud are shown in Fig. 10. Again, one can see a strong redistribution of particles in the flow, but in this case, in contrast to Fig. 9, the narrow layers with high particle concentration disappear. Thus, the polydispersity of the particle phase results in smearing of the concentration field. The physical reason for this effect is mixing of particles of different sizes in the flow. 


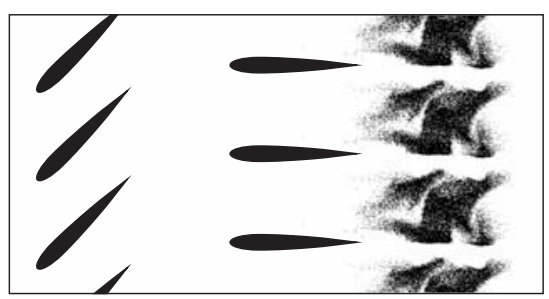

(a)

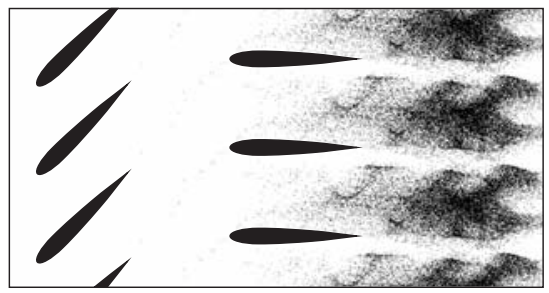

(b)

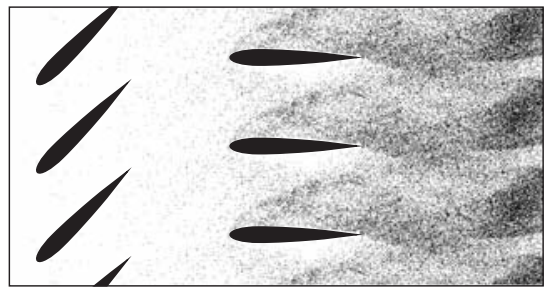

(c)

Figure 10 Instantaneous flow patterns of polydisperse spherical particles (log-normal law of Eq. (4) with $\sigma=1.2$ in an initial cloud) depending on the most probable particle size: $r_{\mathrm{pm}}=5(a), 10(b)$, and $20 \mu \mathrm{m}(c)$; a semiempirical model of particle-wall collisions [11] is used

Consider now the effect of particle scattering in particle-blade collisions due to the nonspherical particle shape and the combined effect of scattering and mixing. In this part of the study, particles are assumed to be solid spheres in the course of motion in the flow, but their shape is transformed to the ellipsoid when they hit blades. To the best of authors' knowledge, the validated collision model for nonspherical particles is absent in the literature. That is why a simplified collision model was used here. The restitution coefficients for the normal $\left(a_{C n}\right)$ and tangential $\left(a_{C \tau}\right)$ velocities of the particle contact point were assumed to be constant and equal to $a_{C n}=0.8$ and $a_{C \tau}=0$.

This means that collisions were considered to be partly nonelastic and nonsliding. This model was also used for spherical particles for the sake of comparison. 


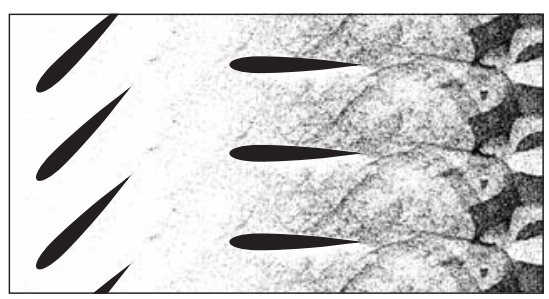

(a)

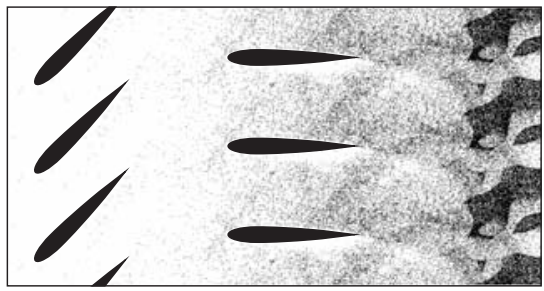

(b)

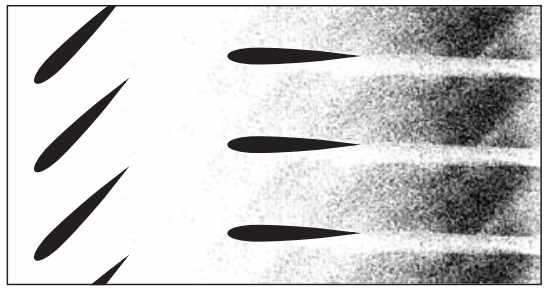

(c)

Figure 11 Instantaneous patterns of particles: monodisperse spherical particles of radius $r_{p}=10 \mu \mathrm{m}(a)$; effect of scattering caused by particle-wall collisions $(b)$; and combined effect of scattering and mixing $(c)$; log-normal law of Eq. (4) with $\sigma=1.2$ is assumed in an initial cloud

Figure $11 a$ shows the results for particles with the shape of prolate ellipsoid (see Fig. $5 a$ ). It is seen from Figs. $11 a$ and $11 b$ that the effect of particle scattering is similar to the effect of particle mixing discussed above: the field of particle concentration becomes smeared. However, the combined effect of scattering and mixing is unexpected at the first glance (cf. Figs. $11 b$ and $11 c$ ). This can be explained by a substantial increase in the number of small particles in the polydisperse mixture. Small particles follow the gas flow more closely than the large ones, and the majority of them do not collide with blades and the effect of scattering decreases.

The role of $3 \mathrm{D}$ effects for particle motion is also worth to be discussed. As was already mentioned, particles do not follow streamlines of the carrier gas due 
to their inertia. In a swirling flow inside the duct of the axial turbomachine, particles have nonzero velocity in the direction normal to the cylindrical stream surface of the carrier gas flow. This results in particle migration towards the duct walls. The displacement of particles after their collisions with blades during the time interval the particles travel between two sequential blades was estimated. For the sake of definiteness, the length of blades was taken equal to $L=1 \mathrm{~m}$ and the number of blades in the rim to $N=36$. Then, the displacement of particles in the radial direction at the input data of the present calculations comprised about $1.5 \%$ of the particle distance from the duct axis and about $10 \%$ of the airfoil chord at a distance of $0.5 \mathrm{~L}$ from the axis. This estimate can serve as a justification of the $2 \mathrm{D}$ flow model used for the particle phase.

\section{CONCLUDING REMARKS}

The present investigation of the dusty gas flow through a rotor-stator set of $2 \mathrm{D}$ cascades showed that dispersed particles were strongly redistributed in the flow. The behavior of the particle phase depended substantially on the particle size. Motion of small particles was governed mainly by the carrier gas flow which was rather complicated due to separation of eddies from blades with forming vortex wakes. Large particles colliding with the blades rebounded from them. The rebounded particles could then collide with both, the nearest and farthest blades, that made the particle phase flow more complicated. In the case of monodisperse spherical particles, narrow layers with high particle concentration arose. Mixing of particles of different size and particle scattering due to nonspherical shape resulted in smearing of these layers. This allows concluding that in actual dusty gas flows, the erosive damage from particle impacts is less than predicted by the classical two-phase flow theory, assuming that the particles are spheres of the same size.

\section{ACKNOWLEDGMENTS}

This study was supported by the Russian Foundation for Basic Research under grant No. 09-08-00888.

\section{REFERENCES}

1. Tabakoff, W., and A. Hamed. 1977. Aerodynamic effect on erosion in turbomachinery. JSME and ASME 1977 Joint Gas Turbine Congress Proceedings. Tokyo, Japan. 574-81. 
2. Stepanov, G. Yu. 1962. Hydrodynamics of cascades of turbomachines. Moscow: FIZMATLIT. [In Russian.]

3. Hussein, M.F., and W. Tabakoff. 1972. Calculation of particle trajectories in a stationary two-dimensional cascade. Dept. of Aero. Engng. TR 72-27, University of Cincinnati, AD-764267.

4. Hussein, M.F., and W. Tabakoff. 1973. Dynamic behavior of solid particles suspended by polluted flow in a turbine cascade. J. Aircraft 10(7):434-40.

5. Tsirkunov, Yu. M. 2001. Gas-particle flows around bodies - key problems, modeling and numerical analysis. 4th Conference (International) on Multiphase Flow. Ed. E. Michaelides. New Orleans, LA, USA. CD-ROM ICMF'2001. 607:31.

6. Anderson, D.A., J. C. Tannehill, and R.H. Pletcher. 1984. Computational fluid mechanics and heat transfer. New York: Hemisphere Publ. Corp.

7. Henderson, Ch. B. 1976. Drag coefficient of spheres in continuum and rarefied flows. AIAA J. 14(6):707-8.

8. Rubinow, S.I., and J.B. Keller. 1961. The transverse force on a spinning sphere moving in a viscous fluid. J. Fluid Mech. 11:447-59.

9. Oesterlé, B., and T. Bui Dinh. 1998. Experiments on the lift of a spinning sphere in a range of intermediate Reynolds numbers. Exper. Fluids 25:16-22.

10. Dennis, S. R. C., S. N. Singh, and D. B. Ingham. 1980. The steady flow due to a rotating sphere at low and moderate Reynolds numbers. J. Fluid Mech. 101:25779.

11. Tsirkunov, Yu.M., S.V. Panfilov, and M. B. Klychnikov. 1994. Semiempirical model of impact interaction of a disperse impurity particle with a surface in a gas suspension flow. J. Engng. Phys. Thermophys. 67(5-6):1018-25.

12. Tsirkunov, Yu., and S. Panfilov. 2007. Particle scattering in particle-wall collisions: The combined effect of the particle non-sphericity and the wall roughness. 6th Conference (International) on Multiphase Flow. Ed. M. Sommerfeld. Leipzig, Germany. CD-ROM ICMF'2007. S2TueA15:10.

13. Panfilov, S. V., and Yu. M. Tsirkunov. 2008. Scattering of non-spherical particles rebounding from a smooth and a rough surface in a high-speed gas-particle flow. J. Appl. Mech. Techn. Phys. 49(2):222-30.

14. Roe, P.L. 1981. Approximate Riemann solvers, parameter vector and difference schemes. J. Comput. Phys. 43(2):357-72.

15. Yee, H. C., and A. Harten. 1987. Implicit TVD schemes for hyperbolic conservation laws in curvilinear coordinates. AIAA J. 25(2):266-74.

16. Romanyuk, D. A., and Yu. M. Tsirkunov. 2010. Numerical simulation of unsteady dusty gas flow through the movimg and stationary cascades of airfoils. 5th Eouropean Conference on Computational Fluid Dynamics: CD-ROM Proceedings. Eds. J. C. F. Pereira and A. Sequeira. Paper No. 1063. 